Original Research

\title{
Mental Health Effects of COVID-19 Pandemic on Healthcare Workers
}

\author{
Sharon F. Clevenger*
}

Indiana Center for Cognitive and Behavioral Therapy, Purdue University, Psychology \& Biology, 423 Airport North Office Park, Fort Wayne, Indiana, USA; E-Mail: sfclevenger@iccbt.org

* Correspondence: Sharon F. Clevenger; E-Mail: sfclevenger@iccbt.org

Academic Editor: Sok Cheon Pak

Special Issue: Complementary, Traditional, and Integrative Medicine for COVID-19

OBM Integrative and Complementary Medicine 2021, volume 6 , issue 1

doi:10.21926/obm.icm.2101004
Received: December 20, 2020

Accepted: January 31, 2021

Published: February 07, 2021

\begin{abstract}
Healthcare workers (HCWs) have been adapting to the changing needs of people worldwide in response to the COVID-19 pandemic. The purpose of this descriptive study was to evaluate how HCWs are managing dietary self-care needs during the increased stress-load of the COVID-19 pandemic and determine if nutritional changes are being made that could place them at higher risk for mental health problems. A cross-sectional epidemiological nonprobability snowball sampling design was used to expand access to participants. Occupational stress is a major concern among HCWs in general; however, increased chronic levels of stress during the COVID-19 pandemic had not been evaluated in terms of stress and dietary behavior as risk factors for mental health problems in this population. The results from this survey indicate that HCWs treating patients during the COVID-19 pandemic are experiencing chronically increased stress-loads, and the increased stress-load is negatively affecting dietary behaviors. Dietary behavior changes included perceived consumption of a less healthy diet overall, increased consumption of sweets and alcoholic drinks, and decreased consumption of fresh fruits and vegetables since the pandemic began. Unhealthy dietary behaviors were associated in a predictive manner with negative mood states in HCWs. In addition, this study uncovered evidence of exhaustion, symptoms of post-traumatic stress disorder and suicidal thinking in this normally resilient population.
\end{abstract}

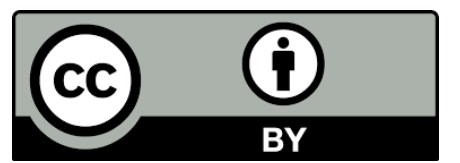

(C) 2021 by the author. This is an open access article distributed under the conditions of the Creative Commons by Attribution License, which permits unrestricted use, distribution, and reproduction in any medium or format, provided the original work is correctly cited. 


\section{Keywords}

Coronavirus; SARS-CoV; mental health; trauma, resilience; healthcare worker; pandemic; nutrition

\section{Introduction}

The National Institute for Occupational Safety and Health (NIOSH) defines occupational stress as "harmful physical and emotional responses that occur when requirements of the job do not match capabilities, resources, or needs of the worker" [1]. According to the Centers for Disease Control (CDC), healthcare workers (HCWs) work in high-stress conditions associated with higher rates of psychological distress than many other occupations. HCWs stressors include work overload, time pressures, lack role clarity for dealing with infectious diseases and difficult, ill and/or helpless patients. Such stressors are known to lead to physical and psychological symptoms, absenteeism, turnover, and medical errors [1]. Burnout in HCWs is frequently associated with poor-quality care and decreased satisfaction in work-life integration in the published literature; however, the actual impact has been difficult to measure because studies were primarily observational limiting conclusions that can be made for causality and directionality $[2,3]$.

\subsection{Progression of COVID-19 as a Pandemic}

Severe Acute Respiratory Distress - Coronavirus (SARS-CoV) emerged from China in 2002 and spread to 26 countries within a few months affecting more than 8,000 people and killing 774 . Coronaviruses are known to "jump" from animals to humans causing pneumonia-like illnesses. Coronaviruses reemerge regularly, and the current strain (COVID-19) reported to the World Health Organization (WHO) in December 2019, has spread rapidly throughout the world [4-6]. On January 30,2020 , the outbreak included 7818 confirmed cases in 18 countries, and two months later, there were 43,103 confirmed cases in 24 countries and 1018 deaths [7]. COVID-19 is associated with severe morbidity and high mortality rates, particularly in older individuals [8]. HCWs treating patients diagnosed with COVID-19 have reported significantly increased stress attributed to an unremitting deluge of ill patients, employment of strict biosecurity measures often without sufficient personal protective equipment (PPE), and additional physical burdens including dehydration, heat, exhaustion, physical isolation (restrictions on touching others, even after working hours), constant vigilance regarding infection control procedures and fear of disease transmission [9]

\subsection{The Stress-Response System}

Chronic or acute exposure to stress is defined as an ongoing or anticipated threat to homeostasis or well-being. Chronic stress-loads favor destabilization of the dynamic balance of the organism, and the stress response system promotes release of chemical mediators that affect metabolic and behavioral states in humans. Upon exposure to a stressor, activation of the neuroendocrine hypothalamic-pituitary-adrenal axis (HPA) exerts widespread metabolic effects necessary to maintain or restore homeostasis to the organism [10]. Stress occurs when homeostasis is 
threatened or perceived to be threatened so the organism strives to re-establish homeostasis through various physiological and behavioral adaptive responses. The stress response system of the body deploys fight-flight neurochemical groups of catecholamines such as epinephrine and norepinephrine, along with cortisol to prepare the organism for defensive and evasive actions. While the catecholamines prepare the body for action, cortisol is released to mobilize energy reserves (glucose) and other substances to provide fuel for that action [11]. The HPA axis as part of the stress response system is composed of complex sets of feedback interactions between the hypothalamus, pituitary gland, and adrenal glands. Neurochemical interactions between these organs create a system of responses intended to prepare the body for fight or flight. Processes that are regulated through the neuroendocrine system direct body processes such as digestion, immune function, mood and emotions, sexuality, energy storage and expenditure. Systems that are unnecessary for fight-flight assist the neuroendocrine system in controlling physiologic and psychologic system activation reactions. The numerous stress response system chemicals that are mobilized target effectors including those regulating executive and/or cognitive functions, reward and fear systems, sleep-wake centers of the brain, thyroid, growth and reproductive hormone axes, and the gastrointestinal, cardiorespiratory, metabolic, and immune systems [12]. Well-known high chronic stress-load-related disorders, such as depression and PTSD, are accompanied by glucocorticoid imbalances and structural/ functional alterations in limbic circuits that resemble those seen in cases of chronic stress. Alteration of limbic and stress effector pathways suggest that inappropriate processing of stressful information may be part of the pathological process that leads to mood dysregulation disorders such as depression and/or anxiety [13].

\subsection{Chronic Stress and Nutrition}

Exposure to high stress-loads is known to modify dietary behaviors toward high calorie hyperpalatable foods [10]. Modification of dietary behaviors under conditions of chronic high stress to high-fat, high-sugar, nutrient poor foods negatively influences neural circuits regulating motivation, emotion, and mood. Resultant alterations of emotion-reward neurobiological pathways occurs influencing energy metabolism, endocrine and immune system function imparting significant systemic biologic stress $[10,14]$. Consumption of certain nutrients is known to be essential for healthy neurobiological function; however, research assessing antecedents contributing to nutritional deficiencies has been lacking, especially in the population of HCWs.

Chronic hyperactivation of HPA axis triggers homeostatic and non-homeostatic pathways that create a pro-obesity biologic environment as suppression of corticotrophin releasing hormone (CRH), leptin resistance and increased hypothalamic neuropeptides such as neuropeptide Y (NPY) stimulate food intake and storage of glucose into storage forms of energy that are diverted into adipose tissues. Neurochemicals are then released that stimulate pathways associated with foodrelated reward and pleasure (dopaminergic and opioidergic pathways) inducing a shift toward a hypercaloric diet that biologically was intended as preparation for long battles or journeys [15]. High cortisol levels raise blood glucose levels which leads to increased insulin secretion, and eventually cells develop insulin resistance. The pancreas compensates for insulin resistance by increasing production of insulin [12]. 


\subsection{Nutrition and Brain Health}

There are known associations between dietary behaviors and the development of healthy brain structure. Healthy brain structure is critical for optimal brain function including cognition, mood, and analytical processing. The brain requires glucose and omega-3 ( $\omega 3)$ polyunsaturated fatty acids ( $\omega 3$-PUFAs) to produce energy. Uptake of glucose by nervous tissue requires the presence of vitamin B1 (thiamine). Chronic deficiency of thiamine is known to cause Wernicke's encephalopathy and Korsakoff's dementia, associated with the development of focal neuronal loss in vulnerable areas of the brain. Thiamine is also a known factor in modulation of cognitive performance, especially in the elderly [16]. Vitamin B9 (folate) deficiency in the periconceptional period contributes to the development of fetal neural tube defects, and deficiencies of vitamin B12 (cobalamin) has negative consequences on the developing brain during infancy. Deficiencies of both folate and cobalamin are associated with a greater risk of developing depression during adulthood [17]. Hyperhomocysteinemia is sometimes associated with functional deficiencies of folate and cobalamin and is responsible for toxicity in neural cells by activating $N$-Methyl- $d$-aspartate (NMDA) receptors [18]. NMDA activity mimics the activity of the excitatory neurotransmitter glutamate. Glutamate is also an excitotoxin that can destroy central nervous system (CNS) neurons by excessive activation of excitatory receptors on dendritic and somal surfaces. Increasing levels of NMDA receptor activation is associated with eventual hypofunction in areas of the brain associated with memory and learning [19].

Tryptophan, an essential amino acid nutrient present in nuts, seeds, cheese and other proteins is the precursor chemical for the neurotransmitter serotonin. Administration of tryptophan may have a mild antidepressant effect in mild-to-moderate clinical depression and may decrease sleep latency in people with mild insomnia, both effects are assumed to be related to an increase in brain serotonin [20]. Vitamin B9 (folate) preserves brain during fetal development and memory as one ages. Vitamin B6 is likely to benefit in treating premenstrual depression. Vitamins B6 and B12, among others, are directly involved in the synthesis of some neurotransmitters. The brain, specifically the nerve endings, contain the second highest concentrations of vitamin C (ascorbic acid) in the human body since the suprarenal glands [21].

People who live in areas with little sunlight during the winter develop an extreme form of seasonal depression manifested as seasonal affective disorder (SAD). SAD is associated with carbohydrate craving, hypersomnia, lethargy, and changes in circadian rhythms. Seasonality and symptoms of SAD may be due to changing levels of vitamin D3 from sunlight, along with shift-work disorder which manifests as a disruption in the circadian rhythm of individuals who like HCWs often work long hours during normal sleep times. Both deficiencies of Vitamin D3 and circadian rhythm disorders are thought to be due to gradual decline of brain serotonin [21]. Vitamin D is a seco-steroid hormone with multiple functions in the nervous system that has been studied due to the role it plays as a nerve hormone in the prevention of various neurodegenerative or neuroimmune diseases [22].

Of the various forms of vitamin $E$ (tocopherols and tocotrienols), only the alpha-tocopherol form is actively taken up by neurons in the brain and is directly involved in neuroprotection. Iron is necessary to ensure oxygenation of the blood, and for the synthesis of neurotransmitters and myelin. Iron deficiencies are commonly found in children who exhibit attention-deficit/hyperactivity symptoms. Magnesium plays an important role systemic metabolism including oxidation-reduction 
reactions and ionic regulation. It is also hypothesized that copper deficiency could be linked to Alzheimer disease. lodine is essential for synthesis of thyroid hormones and also ensures neuronal energy metabolism. Nutrient deficiencies of iodine are known to be associated with severe cerebral dysfunction during pregnancy leading to clinical cretinism. Minerals such as manganese, copper, and zinc participate in enzymatic mechanisms that protect against free radical damage. Brain diseases can be related to failure of the body's normal protective mechanisms that provide for synthesis and deployment of anti-oxidants and individual nutrients and micronutrients are associated with both brain and body health [21].

Dietary consumption of omega-3 ( $\omega 3$ ) polyunsaturated fatty acids ( $\omega 3$-PUFAs) are one of the best-studied interactions between food and brain evolution with docosahexaenoic acid (DHA) being the most abundant $\omega 3$-PUFA in neuronal cell membranes in brain tissues. However, the human body is not efficient at synthesizing DHA, so we are largely dependent on dietary DHA $[23,24]$. The central nervous system (CNS) has the highest concentration of lipids in the human body since adipose tissue. Of the lipid-rich organs, the brain is particularly enriched with polyunsaturated fatty acids (PUFAs) mostly represented by omega-6 $(\omega 6)$ and omega-3 ( $\omega 3)$ series. DHA is the PUFA most needed by the brain, and it assists in helping other PUFAs enter into the brain. Reduced dietary consumption of the $\omega$ - PUFAs (as in the typical Western diet) has been associated with brain diseases, including depression and anxiety [25]. These observations have been supported in the literature, specifically with a meta-analysis by Lin et al. [26] showing significantly lower levels of EPA, DHA, and total w3PUFAs among 3,318 depressed patients. In some of the studies analyzed severity of depressive and anxious symptoms were negatively correlated with blood levels of total w3-PUFA [26].

\subsection{Stress-load, COVID-19 and Mental Health}

There is a known relationship between chronic stress and changes in dietary behaviors; however, very little is known about how these factors interact in HCWs and whether those changes place HCWs at greater risk for mental health problems. The purpose of this study was to evaluate how HCWs are managing dietary self-care needs during the increased stress-load of the COVID-19 pandemic and determine if nutritional changes were being made that could place them at higher risk for mental health problems. Ideally, HCWs must be in good health to meet the demands of their jobs and patients; however, the pandemic has increased already high stress-loads. Chronic exposure to stressful environments and/or stimuli wears out "fight-flight" stress-adaptive processes, weakens the immune system, and increases susceptibility to all-cause disease [27]. High stress-loads are associated with physical and psychological symptoms creating increased financial burden on health care agencies due to worker absenteeism, increased turnover and medical errors [2].

\subsection{Significance of the Current Study}

HCWs affected by chronic high stress-load, the resultant burnout syndrome or physical illnesses may be unable to provide quality healthcare services, take sick leave, and/or change jobs or careers [1]. Health care research is needed to study self-care related antecedents such as dietary behaviors that may affect HCW mental health. Identified antecedents can then be addressed to prevent negative outcomes including severe situational stress (burnout), depression, anxiety, and physical illnesses that may negatively affect patient care, and increase financial costs of replacing and training replacements [28]. 
This research should shed light on mental health risk factors among HCWs during the COVID-19 pandemic providing an essential foundation for planning future prevention strategies that if implemented could mitigate burdens carried during this and future health crises [29]. This study examined dietary self-care behaviors practiced by HCWs during the chronically elevated stress-load of the COVID-19 pandemic. The findings of this study will contribute information to the literature about how HCWs respond to increased stress-loads during public health emergencies, expand on existing knowledge and suggest development of future research to protect this critical workforce during health crises.

\subsection{Healthcare Worker Stress and Burnout}

The National Institute for Occupational Safety and Health (NIOSH) defines occupational stress as "the harmful physical and emotional responses that occur when the requirements of the job do not match the capabilities, resources, or needs of the worker" [1]. The Centers for Disease Control (CDC) has reported that HCWs under normal conditions work in extremely stressful environments that are associated with higher rates of physical and psychological distress than many other occupations. Chronic exposure to stressful environments and/or stimuli, is referred to as "allostatic load" (AL). Chronic allostatic stress-loads or simply "stress-load", may wear out the body's "fight-flight" adaptive regulatory systems. The result of chronic, excessive, stress-load results in alterations in the stress adaptive processes, weakening the system and increasing susceptibility to all-cause disease [13].

According to the transactional theory of stress, stress-load is based on the individual persons perception of stress rather than the impact of any one or more given stressor [19]. Lazarus and Folkman [30] defined stress as the perception of internal forces and the resultant strain on the organism. The term "load" refers to external forces that override the organism's capabilities for homeostasis creating increased demand on the system. Essentially, psychological stress-load refers to the perceived relationship between the person and the environment as one that exceeds their resources and endangers their well-being. There are two phases proposed by Lazarus and Folkman, cognitive appraisal, and coping appraisal. Cognitive appraisal refers to the fact that the stressor has to be cognitive evaluated as potentially threatening, or stressful. Coping appraisal refers to the cognitive and behavioral efforts to master, reduce, or tolerate the external demands created by the stressor. It includes the perceived confidence in one's ability to cope with the situation because one has the resources to cope with it [19].

According to the Yerkes-Dodson Law, performance increases when a person is stressed until the resources of that person are exceeded, and then performance decreases. Stressors that HCWs are exposed to include frequent work overload, time pressures, and often a lack of role clarity for dealing with infectious diseases and difficult, ill, and/or helpless patients. Such stressors are known to lead to physical and psychological symptoms and increased financial burden to health care settings due to absenteeism, turnover, and medical errors [1].

\subsection{Rapidity of Transmission of COVID-19}

In June 2020 when this survey began, the WHO estimated 10 million cases and 500 thousand deaths from COVID-19 globally. By October 29, 2020 there were more than 45 million cases of COVID-19 documented worldwide and 1.2 million deaths. A study in March 2020 examining rates of 
infection in HCWs found that compared with the general community, front-line HCWs were at increased risk for COVID-19 (adjusted HR 11.61, 95\% Cl 10.93-12.33) due to close personal exposure to patients with infections. Estimates suggest HCWs could account for $10-20 \%$ of all cases of infection [31].

\section{Materials and Methods}

Data collection was conducted June 3 through July 15, 2020 using an electronically distributed questionnaire of the target population of licensed HCWs working during the pandemic. This descriptive study assessed levels of HCW perceived stress and dietary behaviors before, since and during the COVID-19 pandemic. The study included a correlational component to examine associations between dietary behaviors and risk of mental health disorders.

\subsection{Survey Construction}

An internet search was conducted for access to licensed HCWs including, but not limited to LISTSERV's, groups and social media pages for nurses (basic and advanced practice), physicians, physician assistants, pharmacists, technicians, and emergency responders. A cross-sectional epidemiological nonprobability snowball sampling design was chosen to expand participation. A survey questionnaire was developed by accessing clusters of items from existing instruments found to be valid and reliable based on the literature. Instruments from which items were adapted included the Pennington Biomedical COVID-19 Survey (PBCS), [32] the Perceived Stress Survey (PSS) [33], the Primary Care PTSD Screen for DSM-5 (PC-PTSD-5) [34, 35], the Patient Health Questionnaire-2 (PHQ-2), the Patient Health Questionnaire-9 (PHQ-9) [36], the Rapid Eating Assessment for Participants - Shortened Version (REAPS) [37], and the National Nurse's Health Study-2 (NHS-2) [38]. Three survey items were added by the investigator to examine additional pandemic-related experiences including: "how do you think your eating habits have changed compared to before the COVID-19 outbreak", "In the past 7 days, how much sleep did you average per 24-hour period?" and "How would you rate your overall sleep quality?" The final survey questionnaire was examined by expert reviewers in the field and members of the investigator's dissertation committee.

\subsection{Participant Protection Methods}

Prior to beginning data collection, the investigator completed National Institutes of Health Protection of Human Subjects in Research training, and approval was obtained from the Huntington University of Health Sciences institutional review board (IRB). Participants reviewed informed consent and were randomly assigned a unique identification number through the software engine "SurveyMonkey" (San Mateo, CA, US), a program compliant with United States Health Insurance Portability and Accountability Act (HIPAA) privacy laws. Access to respondent data was highly restricted granted only to the investigator.

\subsection{Data Collection}

The 60-item survey questionnaire was uploaded to SurveyMonkey, and a weblink generated. The weblink was distributed electronically to a variety of HCWs through professional LISTSERV's and 
social media groups (LinkedIn, Twitter and Facebook). Participants were asked to share the survey weblink with colleagues to broaden the pool of potential respondents. Data from completed questionnaires were downloaded from SurveyMonkey into IBM Corp SPSS, Version 26 statistical software (SPSS) [39]. The raw SPSS dataset was examined and "cleaned" prior to analysis to ensure clear, consistent and readable information [40]. Data cleaning included correction of spelling errors (geographical regions and licenses), elimination of responses from non-HCWs. Missing data and categorical responses were coded for accuracy. Responses more than $50 \%$ incomplete in any category, or less than 10 items past demographic information, were eliminated from analysis.

\subsection{Variable Coding and Processing}

Response options were coded to reflect that higher scores were assigned to "less healthy" behaviors. After variable recoding, original and newly created variables were examined to ensure that all original participant response data matched. Scoring was based on normal ordering, or most likely to impact stress or health. Missing responses were eliminated listwise during statistical analysis to avoid data skewing. Paired sample analysis was used to account for any differences in missing items, or dropout throughout survey progression [41]. Responses were analyzed using frequencies and percentages, then measures of central tendency and variability were applied for items with at least three response options to reveal averages and distributions of responses. Analysis of perceived dietary behaviors for items before/since the pandemic was assessed using Wilcoxon Signed Ranks Test (2-tailed) to compare changes in responses. Pearson's Chi Square was applied to dietary behavior data to compare categorical responses. A significance level of $p<.05$ was established. Spearman's rho was applied to ranked data to determine extent of association between stress and dietary behavior that may result in greater health risks. To examine potential changes in relationships between participants' stress levels and dietary behaviors since the pandemic began, the Wilcoxon Signed Rank Test was applied. Ordinal regression analysis was used to determine if stress-load predicts dietary behavior using ranked means for situational stress-load set and ranked means of dietary behavior change.

\section{Results}

This analysis is based on 521 total respondents of which 494 were HCWs, 19 surveys were eliminated as incomplete past demographic questions or the respondent was not working in healthcare during the pandemic leaving a final sample of 475 total HCWs who completed at least $50 \%$ of the survey. It should be noted that given the limitations inherent in electronically distributed surveys, it is not possible to accurately ascertain the percentage of respondents in relation to the total number of survey recipients, therefore, we are not able to determine what percentage of recipients is represented by the final number of responses (475) making overall representation of HCWs overall unknown. Participant age range was 18 to 80 with modal age of 36 to 50 ( $n=199$ of 475 total). The majority of respondents were female (84\%), white/Caucasian (83\%), working fulltime (84\%) and married/cohabitating (74\%). Professional license categories were collapsed for analysis to 6 possible categories. The majority of respondents were nurses [(advanced practice (30\%) or RNs/LPNs (36\%)]. The majority (54\%) lived at home with at least one other adult. Respondents represented 46 specific geographic regions in the United States (U.S.) mostly residing in the Midwest 
(55\%), or the combined South and Northeast regions (29\%) and 4 respondents reside outside of the U.S.

\subsection{Respondent Testing for COVID-19}

Respondents were asked if they had been tested for COVID-19. Those with negative responses were asked why they had not been tested, and affirmative responses were asked about results of testing. Approximately (81\%) of HCWs reported no symptoms (62\%) or tested negative (18\%). Of those not tested, $10 \%$ reported lack of test availability and some (3\%) were not tested even though they were symptomatic.

\subsection{Regression Analysis Stress-Load and Dietary Behaviors}

Following examination of descriptive data, additional analyses were conducted using Wilcoxon Signed Ranks Test, Spearman's rho, and Pearson chi-square tests to assess associations and changes between perceived behaviors before and since the onset of the pandemic regarding stress levels and dietary behaviors. The Wilcoxon Signed-Ranks Test was used to compare weighted ranks of respondents' bivariate item sets that included perceived before and since COVID-19 items. Ordinal regression was used to analyze association between stress-load and dietary behaviors. Analyses were based on "sets" of question items after ensuring all data were coded in the same direction [41].

Stress-load related items included five variable sets: (a) worry, (b) burnout risk, (c) sleep/fatigue, (d) negative mood, and (e) trauma-stress. Items were ranked with highest weight ranked scores associated with higher stress-load. The range of scores for worry set items was 5-15, burnout (range of 8 -33), sleep/fatigue items (range of 5-19), negative mood (range 4-17) and trauma-stress (range 6-12). Item clusters with potential associations between stress-load and dietary behaviors were analyzed in sets of items before/since the pandemic. Mood and sleep-related items were examined in relationship with mean rank of dietary behavior change to assess for possible correlation. Item sets that included psychological stress-load factors of (a) general stress since COVID-19, (b) perceived situational stress, and (c) trauma-stress items were ranked consistently for dietary behavior item sets (ranked both for before and since the pandemic began) and perceived changes that occurred since the pandemic. Items within each stress-load set were then calculated for weighted rank score per respondent by totalling the rank scores and dividing by number of items in the five variable sets. Rank scores for each set were analyzed to create a mean of rank scores and sum of rank scores for purposes of analysis. Mean rank scores between sets of items could then be compared for possible correlation (i.e. mean ranks of dietary change behavior and mean rank scores of sleep/fatigue items). Effect sizes for correlations between each set of mean ranks item sets was calculated by dividing the $z$-score by the square-root of $N$ (total observations) [41].

\subsection{Worry Stressors}

The results show that the majority of respondents worry about their own health related to the pandemic (81\%), 92\% worry about close friends, family and/or colleagues contracting COVID-19, 76\% reported worry they will infect others close to them, and $71 \%$ report worry they will contract COVID. 
Since the pandemic began, HCWs report greater levels of exhaustion (78\%), general stress levels $(87 \%)$, general levels of worry $(78 \%)$ and sadness $(59 \%)$.

\subsection{Perceived Situational Stressors}

Items in the perceived situational stress-load set were examined for burnout-risk, including decreased confidence in ability to cope, irritability, cynicism, feeling overwhelmed and perceived lack of control over important things. HCWs responses to items assessing burnout, as shown in Table 1 , indicate that most have been upset by something in their work situation (50\%); however, most have confidence in their ability to cope (64\%). Approximately a third reported feeling an inability to control important things often (37\%) or at least some of the time (35\%). Approximately $25 \%$ reported feeling like they cannot cope with everything they have to do, $40 \%$ have often felt angered because of things outside of their control, 26\% reported feeling overwhelmed often, and $44 \%$ reported feeling irritable, cynical, or negative often since the pandemic began.

Table 1 Perceived Situational Stressors (Burnout Risk) in HCWs Since the Onset of the Pandemic.

\begin{tabular}{|c|c|c|c|c|}
\hline Item Associated with Burnout Risk & $f(\%)$ & $\mathrm{N}$ & Mean & $\begin{array}{l}\text { Std. } \\
\text { Deviation }\end{array}$ \\
\hline In general, I feel more stressed. & & 474 & 4.223 & .8269 \\
\hline Strongly agree & $196(41)$ & & & \\
\hline Somewhat agree & $217(46)$ & & & \\
\hline Neutral & $39(8)$ & & & \\
\hline Somewhat disagree & $17(4)$ & & & \\
\hline Strongly disagree & $5(1)$ & & & \\
\hline I have been upset because of something that happened. & & 455 & 2.631 & .9197 \\
\hline Never or almost never & $45(10)$ & & & \\
\hline Sometimes & $172(36)$ & & & \\
\hline Fairly often & $144(30)$ & & & \\
\hline Very Often & $94(20)$ & & & \\
\hline I feel confident about my ability to cope with problems. & & 452 & 2.166 & .8791 \\
\hline Never or almost never & $34(7)$ & & & \\
\hline Sometimes & $116(24)$ & & & \\
\hline Fairly often & $193(41)$ & & & \\
\hline Very often & $109(23)$ & & & \\
\hline I feel less able to control important things. & & 455 & 2.299 & 1.0060 \\
\hline Never or almost never & $112(24)$ & & & \\
\hline Sometimes & $165(35)$ & & & \\
\hline Fairly often & $108(23)$ & & & \\
\hline Very often & $70(15)$ & & & \\
\hline I cannot cope with everything I have to do. & & 455 & 1.993 & 1.0141 \\
\hline Never or almost never & $178(38)$ & & & \\
\hline Sometimes & $159(34)$ & & & \\
\hline
\end{tabular}


Fairly often

Very often $57(12)$

I have felt angered because of things happening outside of my control. $455 \quad 2.424 \quad 1.0401$

Never or almost never $94(20)$

Sometimes $170(36)$

Fairly often $95(20)$

Very often $96(20)$

I have felt like difficulties are piling up and I cannot overcome them. $455 \quad 1.956 \quad 1.0590$

Never or almost never $204(43)$

Sometimes $128(27)$

Fairly often $62(13)$

Very often $61(13)$

I have felt more irritable, cynical, or negative. $454 \quad 2.5199 \quad 1.0583$

Never or almost never

Sometimes $160(34)$

Fairly often $97(20)$

Very often $112(24)$

\subsection{Sleep and Fatigue}

Participants reported worsened sleep (51\%), increased exhaustion (78\%), and likelihood of dozing (51\%) since the pandemic began. Many HCWs reported worsened sleep patterns averaging less than 6 hours of sleep (39\%) and overall sleep quality rated bad or very bad (29\%).

\subsection{Negative Mood States: Depression and Suicidal Thinking}

The effect of perceived situational stress-load on mood since onset of the pandemic was examined by asking how the HCW has felt in the prior two weeks. Results show that HCWs ( $n=453)$ are experiencing negative mood states feeling lack of interest or pleasure for several days (41\%) or more than half of the days (14\%), feeling down, depressed, or hopeless several days (41\%) or more than half of the days (14\%). Approximately $12 \%$ of HCWs reported feeling they would be better off dead several days (10\%), or more than half of the days (2\%).

\subsection{Complex Trauma and Post-Traumatic Stress}

An examination of trauma-related stress during the pandemic uncovered high rates of trauma stress in HCWs. While 35\% acknowledged having experienced a traumatic situation(s) since the pandemic began that included fear, trauma, helplessness and feeling overwhelmed, more than $40 \%$ reported trauma-stress symptoms such as nightmares or upsetting intrusive thoughts. Many (41\%) were using avoidance behaviors if reminded of upsetting events, and $41 \%$ reported experiencing hypervigilance reactions. Nearly half (46\%) reported feeling numb or detached from others or surroundings (dissociated), and $30 \%$ reporting feeling guilt or blame toward self or others related to upsetting event(s). 


\subsection{Changes in Dietary Behavior}

Respondent perception of dietary behaviors and stress-load were analyzed in sets of items before/since the pandemic began. There was an expected increase in eating more than 4-5 meals per week at home compared to before the pandemic ( $83 \%$ vs 59\%) and eating out (less than $2-3$ times per week) ( $35 \%$ vs $83 \%$ before). Alcohol consumption of five or more drinks per week increased from $10 \%$ to $23 \%$. Frequency of usually skip meals increased marginally from $25 \%$ to $30 \%$ since the pandemic. Approximately half of the respondents reported less healthy diet choices in general (53\%), often consuming sweets since the pandemic started (48\%) compared to $28 \%$ before COVID-19. Often use of fried foods increased to $14 \%$ (vs $8 \%$ ), frequent use of sugared beverages doubled from $13 \%$ to $24 \%$. Frequent consumption of fast food increased marginally from $13 \%$ to $16 \%$.

Analysis of perceived dietary behaviors before/since the pandemic was assessed using the Wilcoxon Signed Ranks Test (2-tailed). Results show that overall dietary behaviors became less healthy since the pandemic began for all items assessed. The mean ranks and sums of ranks were calculated for dietary behavior items rated before and since the pandemic began. Scores were compared using the Wilcoxon Paired Signed Ranks Test (2-tailed). Results indicate that dietary behaviors before $\left(M d n=2.25, T_{-}=173.31, S D=.38413\right)$ were perceived to have negatively changed significantly since the start of the pandemic $\left(M d n=2.38, T_{+}=197.2, \mathrm{SD}=.47848\right) \mathrm{W}=4.7, z=-4.939$, $p=<.001, r=0.227$.

\subsection{Analysis Based on Gender and Marital Status}

Dietary behaviors by gender was assessed using Pearson's chi-square statistic based on respondent recall showing differences between genders. Males reported that since the pandemic they have worsened dietary behaviors overall $\left(X^{2}=9.51\right.$ [4], $\left.p=.049\right)$ and significantly greater use of 7 or more alcoholic beverages per week ( $40 \%$ vs $9 \%$ of females) since the pandemic ( $X^{2} 51.404$ [8], $p$ $=<.001$ ). Males reported decreased consumption of fresh fruits and vegetables (64\%) compared to females $(29 \%)$ since the pandemic $\left(X^{2}=22.185\right.$ [4], $\left.p=<.001\right)$. No differences were found in dietary behaviors before and since the pandemic based on marital status or living alone $(n=463)$.

\subsection{Dietary Change, Mood and Sleep}

Mood and sleep-related items were examined in relationship with mean rank of dietary behavior change using Spearman's rho correlation test. Response options for each item were assigned weights compiled and ranked for item sets (i.e. dietary behavior change, worry, burnout risk, negative mood, sleep/fatigue, and trauma stress). Reported hours of sleep were correlated with mean ranks of dietary behavior change using Spearman's rho correlation. Of the 181 out of 441 respondents who reported getting less than six hours of sleep, less sleep was associated with change to unhealthier dietary behaviors $(n=210)$ ( $r$ ho $=.189, p<.001)$. Mean ranks of dietary behavior change were correlated with mean ranks for stress, worry, trauma, sleep/fatigue, and mood items. Table 2 summarizes the results of the Spearman rho correlation test for each of these items showing significant positive association between mean ranks sets of stressors and mean ranks change in dietary behaviors $(p<.001)$ for each stressor to worsened dietary behaviors $(N=465)$. 
Table 2 Association of Dietary Behavior Change with Worry, Burnout, Negative Mood, Sleep/Fatigue and Trauma-stress.

Mean Ranks Dietary Behavior Change Items Spearman's rho Correlation ${ }^{a}$

By Mean Ranks Stress Set of Items

\begin{tabular}{ll} 
Worry & .218 \\
Situational Stress (Burnout Risk) & .304 \\
Negative Mood & .377 \\
Sleep/fatigue & .288 \\
Trauma Stress & .292 \\
\hline
\end{tabular}

Note: $\mathrm{N}=465 ;{ }^{\mathrm{a}}$ All measures were significant at $p<.001$

\subsection{Directional Analysis: Stress, Diet Change and Mood}

Responses for psychological stress-load factor item sets for (a) general stress since COVID, (b) perceived situational stress, and (c) trauma-stress were compared with dietary behavior item sets ranked both for before and since behaviors and perceived changes that occurred since the pandemic. The Wilcoxon Signed Rank Test for association between perceived worsened dietary behavior change and actual recalled dietary behavior change was not significant indicating that $\mathrm{HCWs}(\mathrm{N}=465)$ were likely to be accurate in describing their dietary behaviors ( $W=44328.50, z=-1.721, p=.085$, $r=.0798$ ). Those reporting highest stress scores also reported greater frequency of worsened dietary behaviors since COVID ( $n=220$ of 465). Mean ranks of Strongly Agree for more stressed in general since COVID and mean ranks of Worsened dietary behaviors since COVID were significantly correlated in a positive direction $(n=195$, rho $=.257, p<.001)$. Correlation between mean ranks of Worsened dietary change and mean rank score for situational stress item set (scores greater than 3.25 out of 5.00$)(n=85, r h o=.304, p<.001)$ and mean rank scores for high trauma-stress (scores greater than 1.75 out of 2.00$)(n=103, r h o=.292, p<.001)$ were also significantly correlated in a positive direction as shown in Table 3.

Table 3 Correlation Between Stressor by Response of Worsened Dietary Behaviors Since COVID.

\begin{tabular}{lllll}
\hline $\begin{array}{l}\text { Worsened Dietary Behavior Since COVID } \\
(n=220) \text { by Stressor (Score) }\end{array}$ & $\mathrm{f}(\%)$ & Spearman's rho $^{\mathrm{a}}$ & Total N & Score Range \\
\hline Strongly agree more stressed (5) & $195(42)$ & .257 & 465 & $1-5$ \\
Mean ranks Situational Stress (>3.25) & $85(20)$ & .304 & 465 & $1-5$ \\
Mean ranks Trauma-Stress (>1.75) & $103(23)$ & .292 & 446 & $1-2$ \\
\hline
\end{tabular}

Note: Mean ranks of Worsened Dietary Behavior $n=220$ of $465 .{ }^{a}$ All items were significant at $p$ $<.001$.

\subsection{Regression Analysis: Stress-Load and Dietary Behavior Change}

An ordinal regression analysis was used to determine if stress-load predicts dietary behavior using ranked means for the situational stress-load set and ranked means of dietary behavior change. Model fitting showed that the Pearson chi-square test $\left[X^{2}(29)=87.150, p<.001\right]$ was significant, and 
the Goodness of Fit model $\left[X^{2}(145)=172.906, p=.057\right]$ was non-significant, both suggesting that the data are a good fit for the model. The explanatory variable of situational stress improved the model because unexplained variation decreases from 521.603 in the model with only a constant, to 434.452, difference of 87.150 which is statistically significant $(p<.001)$. The proportional odds model shows a positive effect $b=2.883$ (95\% Cl 0.167-5.599), which is statistically significant according to the Wald test $(p=.038)$. The Nagelkerke Pseudo R-Square $(0.176)$ indicated that $17.6 \%$ of the variance of the model is explained by interaction of the two variables. There is also evidence of association between the two variables based on analysis of the slope. The test of parallel lines shows that the -2 Log Likelihood of the curves have the same slope $\left[X^{2}(145)=170.700, p=.071\right]$ indicating that the null hypothesis can be rejected. The results of the analysis therefore shows an association between the variables that stress-load is predictive of dietary behavior change, and that HCWs with higher stress-loads and worsened dietary habits experienced increased negative mood states since the pandemic began.

\section{Discussion}

HCWs treating patients diagnosed with COVID-19 have reported significantly greater stress levels than usual attributed to employing strict biosecurity measures, often without sufficient PPE, physical burdens of dehydration, heat, exhaustion, physical isolation (restrictions on touching others, even after working hours), constant vigilance regarding infection control procedural pressures and fear due to risk of disease transmission [9].

\subsection{Stress-Load Factors in HCWs}

According to the transactional theory of stress, stress-load is based on individual perception of stress rather than the impact of any given stressor(s) [30]. According to Lazarus and Folkman [30] "stress" is defined as the perception of internal forces and resultant strain on the organism. "Load" refers to external forces that override the organism's capabilities for homeostasis creating increased demand on the system [30]. Essentially, psychological stress-load refers to the perceived relationship between person and environment as one that exceeds resources and endangers their well-being. Results of this study revealed five areas of increased stress-load among HCWs related to the COVID-19 pandemic: (a) worry, (b) burnout risk, (c) sleep/fatigue, (d) negative mood, and (e) trauma-stress. Effect of chronic increased stress-load on HCWs is shown through participant responses indicating worsened sleep, exhaustion, and negative mood states since the pandemic began. A troubling finding was that $12 \%$ of HCWs reported feeling like they would be better off dead for several days (10\%), or more than half of the days (2\%). HCWs were not recognizing symptoms of trauma-related stress with $35 \%$ of $\mathrm{HCWs}$ reporting having experienced trauma-stress situations since the pandemic; however, more than 40\% ( $n=103$ of 465 respondents) scored in the "high" range of trauma-stress (>1.75 out of possible 2.00). 40\% reported trauma-stress symptoms of nightmares or upsetting intrusive thoughts, $41 \%$ reported avoidance and hypervigilance behaviors, and nearly half (46\%) reported feeling numb or detached from others or surroundings (dissociated).

Approximately half of the respondents reported having less healthy diet overall since the pandemic began including consuming more alcohol and sweets often. Consumption of fresh fruits and vegetables decreased, more in males compared to females. In general, HCWs are reporting high levels of worry, situational and trauma-stress and exhaustion which correlate with worsened dietary 
behaviors. An ordinal regression analysis uncovered a directional association suggesting that stressload predicts dietary behavior change and that HCWs with higher stress-loads and worsened dietary behaviors experienced increased negative mood states since the pandemic began

\subsection{Psychological Effects of the Pandemic}

Stress related to the COVID-19 pandemic has been associated with anxiety, fear of contagion, depression, insomnia, and social isolation. Social isolation combined with added anxiety and stress risks the development or exacerbation of depression, anxiety and substance use disorders in vulnerable populations including HCWs. Stress related psychiatric conditions including mood and substance use are associated with suicidal thinking and behavior [42], post-traumatic stress disorder (PTSD) and increased incidence of domestic violence [43]. A multicenter, cross-sectional survey of 4,692 nurses in China working during the COVID-19 pandemic found that $9.4 \%$ had symptoms of depression, $8.1 \%$ had anxiety and $6.5 \%$ had suicidal thinking [44]. A second study of $8817 \mathrm{HCWs}$ in China uncovered prevalence of depression (30\%), anxiety (21\%), and suicidal thinking (6.5\%) due to psychological stress during the pandemic [45]. This suggests that suicidal thinking is associated with increased stress during pandemic in numbers similar to the current study which uncovered that $12 \%$ of HCWs reported suicidal thinking.

\subsection{Burnout Risk Related to Stress-Load}

There is high risk for burnout in HCWs under chronic high stress-loads. Symptoms predicting burnout include decreased confidence in ability to cope, irritability, cynicism, feeling overwhelmed and perceived lack of control over important things [46, 47]. Most respondents to the current survey reported confidence in their ability to cope with problems (64\%); however, many reported experiencing other symptoms of burnout risk such as exhaustion or exhaustion risk. Many indicated worsened sleep reporting an average of less than 6 hours of sleep (39\%), and overall sleep quality rated as bad or very bad (29\%).

\subsection{Perception of Trauma-Stress by HCWs}

Respondents reported experiencing symptoms of trauma-related stress (40-46\%); however, fewer reported experiencing trauma (35\%). The discrepancy might be due to the perception by HCWs that responding to, and working in, extremely high-stress situations (i.e. crowded emergency rooms with multiple victims, watching someone die horrifically while being helpless to change the situation, constant hypervigilance about contracting the virus and injuring/infecting others) is just "part of the job" and therefore not perceived as trauma. Some HCWs did not respond to the trauma set of questions ( $n=31$ ). If they failed to respond due to discomfort with the questions or inability to identify or acknowledge their experiences, the number of HCWs suffering from trauma-stress could be higher than what was uncovered in this sample.

\subsection{Chronic High Stress Load Predicts Unhealthy Dietary Behaviors}

The results from this study show that HCWs under chronic high stress-load alter their diet toward unhealthy choices. Gender differences were noted in that males reported worsened diet, greater consumption of alcoholic beverages, and eating fewer fresh fruits and vegetables compared to 
females. Reviews of the literature point toward being single or living alone associated with less healthy dietary habits $[44,46]$; however, this study found no difference in these parameters with regard to snacking, overall diet choice, use of alcoholic beverages or use of fresh fruits and vegetables. The lack of differences could be due to the sample obtained for this study being heavily skewed toward Caucasian female, married, professional nurses. The limited diversity represented as far as ethnic strata may have failed to uncover differences in socially associated dietary behaviors.

\subsection{Unhealthy Dietary Behaviors Increases Risk for Mental Health Problems}

Results of this study suggest that those with increased unhealthy dietary behaviors were more likely to experience increased stress-load and negative mental health symptoms including traumastress, burnout risk, worry, sleep problems, exhaustion, and negative mood. Those who reported less sleep reported more unhealthy dietary behaviors. A direct link between nutrient levels and mood could not be made because biologic samples were not obtained; however, the findings suggest that changes in dietary behaviors occurred related to chronic increased stress-load. Findings strengthen the currently available evidence linking emotional exhaustion, depersonalization and feelings of reduced personal accomplishment combined with changes in dietary behaviors thereby placing this group of HCWs at higher risk of developing mental health problems including depression, anxiety and PTSD $[46,47]$.

Given that respondents were likely to be accurate describing their perceived dietary behavior patterns, the findings that as perceived stress-load increased, so did worsened choices in dietary behaviors suggests that HCWs under increased pandemic stress-load are exhibiting less healthy dietary behaviors. Findings also suggest that stress-load is predictive of changes in dietary behavior, HCWs with higher stress-load had more unhealthy dietary behaviors, and those with more unhealthy dietary behaviors experienced increased negative mood states since the pandemic began.

\subsection{Limitations of the Current Study}

The findings of the current study should be considered given certain limitations uncovered during analysis. There were dropouts as the survey questions progressed with most of early items answered, and more items skipped as the survey progressed. Some participants may have dropped out due questionnaire length, and others that did not participate may represent a completely different population, for example, highly stressed HCWs that did not respond due to the burden of completing a survey. Results obtained were based on a sample of HCWs heavily skewed toward Caucasian female, married, professional nurses with limited diversity as far as ethnicity, gender, and geographic region; therefore, the results may lack important socially-associated differences in dietary behaviors. Given the gender distribution of the final sample, we cannot conclude that the results are representative of HCWs in the U.S. as a whole. The overall percentage of females in the final sample (84\%) is somewhat higher than the percentage of females in national samples of HCWs, which is about $76 \%$; however, given that women make up more than $85 \%$ of workers in nursing, the sample obtained is probably fairly representative of HCWs in the U.S. [48]. 


\section{Conclusions}

Stressors that exist among HCWs that increase stress-load related to the pandemic include worry stress about their own health, contracting COVID-19, about close friends, family and/or colleagues and about infecting others close to them. HCWs are reporting worsened quality of sleep, greater levels of exhaustion, increased general stress levels and general levels of worry and sadness. Burnout risks reported by HCWs included increased irritability, cynicism, feeling overwhelmed, anger over things they cannot control and perceived lack of control over important things. HCWs are reporting consumption of unhealthier diets with more alcohol, higher fat and sugar, and decreased consumption of healthy foods. These unhealthy changes in dietary behaviors in combination with increased chronic stress places HCWs at great risk for mental health problems. Uncovered associations between stress-load and dietary behaviors suggest that (a) stress-load is predictive of dietary change behavior, and (b) HCWs with higher stress-loads and worsened dietary behaviors experienced increased negative mood states since the pandemic began. Due to limitations inherent in an electronic survey, it was not possible to obtain biologic samples from the HCWs who reported higher than normal stress-load. Examination of actual vs hypothetical nutrient content and the effects of social isolation on mental health would be important next steps for research [29].

\subsection{Resiliency Factors, Stress, Diet and Trauma}

The majority of HCWs possess high levels of protective factors that provide resilience against psychological impact of trauma, the results of this study uncovered deterioration of many protective factors including adequate restorative sleep, healthy dietary choices, limited use of alcohol, optimism, perception of control and confidence to cope. HCWs reported significant amounts of trauma-stress symptoms (40-46\%); however, fewer reported exposure to situation(s) considered traumatic such as working conditions during this pandemic (35\%). The discrepancy may indicate a gap in knowledge for recognizing trauma-stress in this population. Future research could examine the temporal basis for changes in sleep, eating behaviors and perceived trauma stressors related to HCWs. This information would be useful for education and training purposes to recognize and intervene for those at risk for trauma disorders and burnout risk, including oneself. It was especially troubling to observe that $12 \%$ of HCWs reported feeling like they would be better off dead for several days (10\%), or more than half of the days (2\%). Measures to identify and intervene with HCWs at risk for suicide is a critical area for future research.

\section{Author Contributions}

Sharon F. Clevenger did all the research work of this study.

\section{Competing Interests}

The authors have declared that no competing interests exist. 


\section{References}

1. Centers for Disease Control. Exposure to stress: Occupational hazards in hospitals. Washington, D.C.: Prevention Department of Health and Human Services, National Institute for Occupational Safety and Health; 2008.

2. Tawfik DS, Scheid A, Profit J, Shanafelt T, Trockel M, Adair KC, et al. Evidence relating health care provider burnout and quality of care: A systematic review and meta-analysis. Ann Intern Med. 2019; 171: 555-567.

3. Dyrbye LN, West CP, Johnson PO, Cipriano PF, Beatty DE, Peterson C, et al. Burnout and satisfaction with work-life integration among nurses. J Occup Environ Med. 2019; 61: 689-698.

4. Wu D, Wu T, Liu Q, Yang Z. The SARS-CoV-2 outbreak: What we know. Int J Infect Dis. 2020; 94: 44-48.

5. Ksiazek TG, Erdman D, Goldsmith CS, Zaki SR, Peret T, Emery S, et al. A novel coronavirus associated with severe acute respiratory syndrome. N Engl J Med. 2003; 348: 1953-1966.

6. Zhu N, Zhang D, Wang W, Li X, Yang B, Song J, et al. A novel coronavirus from patients with pneumonia in China, 2019. N Engl J Med. 2020; 382: 727-733.

7. World Health Organization (WHO). Novel Coronavirus (2019-nCoV): situation report-22 [Internet]. Geneva: World Health Organization; 2020. Available from: https://www.who.int/emergencies/diseases/novel-coronavirus-2019/situation-reports.

8. World Health Organization (WHO). Rolling updates on coronavirus disease (COVID-19) [Internet].int: Geneva: World Health Organization; 2020. Available from: https://www.who.int/emergencies/diseases/novel-coronavirus-2019/events-as-they-happen.

9. Managing healthcare workers' stress associated with the COVID-19 virus outbreak [Internet]. Washington, D.C.: US Department of Veterans Affairs; 2020. Available from: https://www.ptsd.va.gov/covid/COVID healthcare workers.asp.

10. Morera LP, Marchiori GN, Medrano LA, Defagó MD. Stress, dietary patterns and cardiovascular disease: A mini-review. Front Neurosci. 2019; 13: 1226.

11. Tsigos C, Chrousos GP. Hypothalamic-pituitary-adrenal axis, neuroendocrine factors and stress. J Psychosom Res. 2002; 53: 865-871.

12. Chrousos GP. Stress and disorders of the stress system. Nat Rev Endocrinol. 2009; 5: 374-381.

13. Herman JP. Neural control of chronic stress adaptation. Front Behav Neurosci. 2013; 7: 61.

14. Barnard ND, Bunner AE, Agarwal U. Saturated and trans fats and dementia: A systematic review. Neurobiol Aging. 2014; 35: S65-S73.

15. Ouakinin SR, Barreira DP, Gois CJ. Depression and obesity: Integrating the role of stress, neuroendocrine dysfunction and inflammatory pathways. Front Endocrinol. 2018; 9: 431.

16. Hazell AS, Faim S, Wertheimer G, Silva VR, Marques CS. The impact of oxidative stress in thiamine deficiency: A multifactorial targeting issue. Neurochem Int. 2013; 62: 796-802.

17. Black MM. Effects of vitamin B12 and folate deficiency on brain development in children. Food Nutr Bull. 2008; 29: S126-S131.

18. Smach MA, Naffeti S, Charfeddine B, Ben Abdallah J, Othmen LB, Letaef A, et al. Homocysteine, vitamin B-12, folic acid and the cognitive decline in the elderly. Pathol Biol. 2013; 61: 184-192.

19. Newcomer JW, Farber NB, Olney JW. NMDA receptor function, memory, and brain aging. Dialogues Clin Neurosci. 2000; 2: 219-232. 
20. Young SN. Clinical nutrition: 3. The fuzzy boundary between nutrition and psychopharmacology. Can Med Assoc J. 2002; 166: 205-209.

21. Bourre JM. Effects of nutrients (in food) on the structure and function of the nervous system: update on dietary requirements for brain. Part 1: micronutrients. J Nutr Health Aging. 2006; 10: 377-385.

22. Kalueff AV, Tuohimaa P. Neurosteroid hormone vitamin $D$ and its utility in clinical nutrition. Curr Opin Clin Nutr Metab Care. 2007; 10: 12-19.

23. Gómez-Pinilla F. Brain foods: The effects of nutrients on brain function. Nat Rev Neurosci. 2008; 9: 568-578.

24. Simopoulos AP. Dietary omega-3 fatty acid deficiency and high fructose intake in the development of metabolic syndrome, brain metabolic abnormalities, and non-alcoholic fatty liver disease. Nutrients. 2013; 5: 2901-2923.

25. Larrieu T, Layé S. Food for mood: Relevance of nutritional omega-3 fatty acids for depression and anxiety. Front physiol. 2018; 9: 1047.

26. Lin PY, Huang SY, Su KP. A Meta-Analytic Review of Polyunsaturated Fatty Acid Compositions in Patients with Depression. Biol Psychiatry. 2010; 68: 140-147.

27. Seeman TE, McEwen BS, Rowe JW, Singer BH. Allostatic load as a marker of cumulative biological risk: MacArthur studies of successful aging. Proc Natl Acad Sci U S A. 2001; 98: 4770.

28. Ruotsalainen JH, Verbeek JH, Mariné AS. Preventing occupational stress in healthcare workers. Cochrane Database Syst Rev. 2015; 2015: CD002892. doi: 10.1002/14651858.CD002892.pub5.

29. El-Hage W, Hingray C, Lemogne C, Yrondi A, Brunault P, Bienvenu T, et al. Health professionals facing the coronavirus disease 2019 (COVID-19) pandemic: What are the mental health risks? Encephale. 2020; 46: S73-S80.

30. Lazarus RS, Folkman S. Stress, appraisal and coping. New York, NY: Springer Publishing Company; 1984.

31. Nguyen LH, Drew DA, Graham MS, Joshi AD, Guo CG, Ma W, et al. Risk of COVID-19 among front-line health-care workers and the general community: A prospective cohort study. Lancet Public Health. 2020; 5: e475-e483.

32. Redman LM. Pennington biomedical COVID-19 survey [Internet]. Baton Rouge: Pennington Biomedical Research 2020.2 Available from: https://www.pbrc.edu/coronavirus/research/.

33. Cohen S, Williamson $\mathrm{G}$. The perceived stress scale in a probability sample of the United States. In: The social psychology of health: Claremont Symposium on applied social psychology. Newbury Park: Sage; 1988. pp.31-67.

34. Prins A, Bovin MJ, Kimerling R, Kaloupek DG, Marx BP, Pless Kaiser A, et al. The Primary Care PTSD Screen for DSM-5 (PC-PTSD-5) [Internet]. Washington, D.C.: US Department of Veterans Affairs; 2015. Available from: https://www.ptsd.va.gov/professional/assessment/screens/pcptsd.asp.

35. Sveen J, Bondjers K, Willebrand M. Psychometric properties of the PTSD checklist for DSM-5: A pilot study. Eur J Psychotraumatol. 2016; 7: 30165.

36. Kroenke K, Spitzer RL, Williams JB. The patient health questionnaire-2: Validity of a two-item depression screener. Med Care. 2003; 41: 1284-1292. 
37. Sundermann EE, Katz MJ, Lipton RB, Lichtenstein AH, Derby CA. A brief dietary assessment predicts executive dysfunction in an elderly cohort: Results from the einstein aging study. J Am Geriatr Soc. 2016; 64: e131-e136.

38. Nurses' health study. Boston: Harvard University; 2016. Available from: http://www.nurseshealthstudy.org/.

39. IBM Corporation. IBM SPSS statistics grad pack for windows. 26.0 ed. Armonk: IBM Corp; 2019.

40. Morgan GA, Leech NL, Gloeckner GW, Barrett KC. SPSS for Introductory statistics: Use and interpretation. 3rd ed. Mahwah, New Jersey: Lawrence Erlbaum Associates, Publishers; 2007.

41. Field A. Discovering statistics using IBM SPSS statistics. 5th ed. Thousand Oaks, CA: Sage Publications Ltd.; 2018.

42. Sher L. The impact of the COVID-19 pandemic on suicide rates. QJM. 2020; 113: 707-712.

43. O'Neil A, Nicholls SJ, Redfern J, Brown A, Hare DL. Mental health and psychosocial challenges in the COVID-19 pandemic: Food for thought for cardiovascular health care professionals. Heart Lung Circ. 2020; 29: 960-963.

44. Hong $\mathrm{S}, \mathrm{Ai} M, \mathrm{Xu} X$, Wang $\mathrm{W}$, Chen J, Zhang $\mathrm{Q}$, et al. Immediate psychological impact on nurses working at 42 government-designated hospitals during COVID-19 outbreak in China: A crosssectional study. Nurs Outlook. 2021: 69: 6-12.

45. Xiaoming X, Ming A, Su H, Wo W, Jianmei C, Qi Z, et al. The psychological status of 8817 hospital workers during COVID-19 Epidemic: A cross-sectional study in Chongqing. J Affect Disord. 2020; 276: 555-561.

46. Maslach C, Jackson S. The measurement of experienced burnout. J Occup Behavior. 1981; 2: 99-113.

47. Rodrigues H, Cobucci R, Oliveira A, Cabral JV, Medeiros L, Gurgel K, et al. Burnout syndrome among medical residents: A systematic review and meta-analysis. PloS One. 2018; 13: e0206840.

48. Cheesman JD, Christnacht C. America counts: Stories behind the numbers [Internet]. Washington, D.C.: U.S. Department of Commerce; 2020. Available from: https://www.census.gov/AmericaCounts.

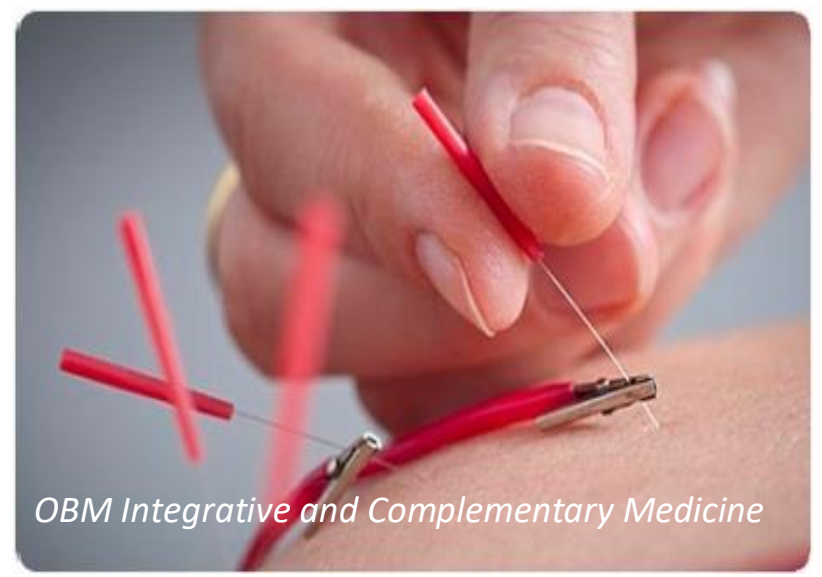

Enjoy OBM Integrative and Complementary Medicine by:

1. Submitting a manuscript

2. Joining in volunteer reviewer bank

3. Joining Editorial Board

4. Guest editing a special issue

For more details, please visit: http://www.lidsen.com/journals/icm 